\title{
Using maple to study the double integral problems
}

\section{Chii-Huei Yu}

Department of Management and Information, Nan Jeon Institute of Technology, Tainan City, Taiwan

\section{Email address:}

chiihuei@mail.njtc.edu.tw (Chii-Huei Yu)

\section{To cite this article:}

Chii-Huei Yu. Using Maple to Study the Double Integral Problems, Applied and Computational Mathematics. Vol. 2, No. 2, 2013, pp. 28-31. doi: 10.11648/j.acm.20130202.12

\begin{abstract}
This paper uses the mathematical software Maple as the auxiliary tool to study the evaluation of two types of double integrals. We can find the closed forms of these two types of double integrals by using Euler's formula and finite geometric series. On the other hand, we propose four examples to do calculation practically. The research methods adopted in this study involved finding solutions through manual calculations and verifying these solutions by using Maple. This type of research method not only allows the discovery of calculation errors, but also helps modify the original directions of thinking from manual and Maple calculations. For this reason, Maple provides insights and guidance regarding problem-solving methods.
\end{abstract}

Keywords: Double Integrals, Euler's Formula, Finite Geometric Series, Closed Forms, Maple

\section{Introduction}

As information technology advances, whether computers can become comparable with human brains to perform abstract tasks, such as abstract art similar to the paintings of Picasso and musical compositions similar to those of Mozart, is a natural question. Currently, this appears unattainable. In addition, whether computers can solve abstract and difficult mathematical problems and develop abstract mathematical theories such as those of mathematicians also appears unfeasible. Nevertheless, in seeking for alternatives, we can study what assistance mathematical software can provide. This study introduces how to conduct mathematical research using the mathematical software Maple. The main reasons of using Maple in this study are its simple instructions and ease of use, which enable beginners to learn the operating techniques in a short period. By employing the powerful computing capabilities of Maple, difficult problems can be easily solved. Even when Maple cannot determine the solution, problem-solving hints can be identified and inferred from the approximate values calculated and solutions to similar problems, as determined by Maple. For this reason, Maple can provide insights into scientific research. Inquiring through an online support system provided by Maple or browsing the Maple website (www.maplesoft.com) can facilitate further understanding of Maple and might provide unexpected insights. For the instructions and operations of Maple, [1-7] can be adopted as references.
In calculus and engineering mathematics curricula, determining the surface area, the volume under a surface, and the center of mass of a lamina requires using double integrals. Therefore, both the evaluations and numerical calculations of double integrals possess significance, and can be studied based on [8-14]. This paper mainly studies the following two types of double integrals

$$
\begin{gathered}
\int_{y_{1}}^{y_{2}} \int_{x_{1}}^{x_{2}} \frac{1-y \cos x-y^{n+1} \cos (n+1) x+y^{n+2} \cos n x}{1-2 y \cos x+y^{2}} d x d y \\
\int_{y_{1}}^{y_{2}} \int_{x_{1}}^{x_{2}} \frac{y \sin x-y^{n+1} \sin (n+1) x+y^{n+2} \sin n x}{1-2 y \cos x+y^{2}} d x d y
\end{gathered}
$$

where $n$ is any non-negative integer, and

$$
x_{1}, x_{2}, y_{1}, y_{2}
$$

are real numbers such that

$$
\left(k \pi,(-1)^{k}\right) \notin\left[x_{1}, x_{2}\right] \times\left[y_{1}, y_{2}\right]
$$

for all integers $k$. These two types of double integrals are related to double integrals studied in [15-20], but our methods are different from the methods used in these papers. The main methods used in [15-20] to determine the double integrals including integration term by term, geometris series, power series expansions, and so on. And the answers of these double integrals obtained are presented in infinite series forms. In this study, we can determine the closed forms of the double integrals (1) and (2) by using the 
Euler's formula and the finite geometric series; these are the main results of this study (i.e., Theorems 1 and 2). On the other hand, we propose four double integral examples to do calculation practically. The research methods adopted in this study involved finding solutions through manual calculations and verifying these solutions by using Maple. This type of research method not only allows the discovery of calculation errors, but also helps modify the original directions of thinking from manual and Maple calculations. Therefore, Maple provides insights and guidance regarding problem-solving methods.

\section{Main Results}

Firstly, we introduce the notations and formulas used in this paper.

\section{Notations:}

Let $z=a+i b$ be a complex number, where $a, b$ are real numbers. We denote $a$ the real part of $z$ by $\operatorname{Re}(z)$, and $b$ the imaginary part of $z$ by $\operatorname{Im}(z)$.

Euler's Formula:

$e^{i x}=\cos x+i \sin x$, where $x$ is any real number.

\section{Finite Geometric Series:}

$$
1+r+r^{2}+\cdots+r^{n}=\frac{1-r^{n+1}}{1-r} \text {, where } r \text { is a real }
$$

number, $r \neq 1$ and $n$ is any non-negative integer.

Before deriving the first result of this paper, we need a lemma.

Lemma 1. Suppose that $x, y$ are real numbers, $n$ is any non-negative integer, and $(x, y) \neq\left(k \pi,(-1)^{k}\right)$ for all integers $k$. Then

$$
\begin{aligned}
& \frac{1-y \cos x-y^{n+1} \cos (n+1) x+y^{n+2} \cos n x}{1-2 y \cos x+y^{2}} \\
& =\sum_{k=0}^{n} y^{k} \cos k x
\end{aligned}
$$

\section{Proof.}

$$
\begin{aligned}
& \frac{1-y \cos x-y^{n+1} \cos (n+1) x+y^{n+2} \cos n x}{1-2 y \cos x+y^{2}} \\
& =\frac{(1-y \cos x)\left[1-y^{n+1} \cos (n+1) x\right]+y^{n+2} \sin (n+1) x \cdot \sin x}{(1-y \cos x)^{2}+(y \sin x)^{2}} \\
& =\operatorname{Re}\left\{\frac{\left[1-y^{n+1} \cos (n+1) x\right]-i y^{n+1} \sin (n+1) x}{(1-y \cos x)-i y \sin x}\right\} \\
& =\operatorname{Re}\left\{\frac{1-y^{n+1} \cdot e^{i(n+1) x}}{1-y \cdot e^{i x}}\right\}(\text { by Euler's Formula) } \\
& =\operatorname{Re}\left\{\sum_{k=0}^{n}\left(y \cdot e^{i x}\right)^{k}\right\}(\text { by Finite Geometric Series ) } \\
& =\sum_{k=0}^{n} y^{k} \cos k x \text { (by Euler's Formula) }
\end{aligned}
$$

The following is our first result, we obtained the closed form of the double integral (1).

Theorem 1. Assume that $n$ is any non-negative integer, and $x_{1}, x_{2}, y_{1}, y_{2}$ are real numbers such that $\left(k \pi,(-1)^{k}\right) \notin\left[x_{1}, x_{2}\right] \times\left[y_{1}, y_{2}\right]$ for all integers $k$.

Then

$$
\begin{aligned}
& \int_{y_{1}}^{y_{2}} \int_{x_{1}}^{x_{2}} \frac{1-y \cos x-y^{n+1} \cos (n+1) x+y^{n+2} \cos n x}{1-2 y \cos x+y^{2}} d x d y \\
& =\left(x_{2}-x_{1}\right)\left(y_{2}-y_{1}\right)+ \\
& \sum_{k=1}^{n} \frac{1}{k(k+1)}\left(\sin k x_{2}-\sin k x_{1}\right)\left(y_{2}{ }^{k+1}-y_{1}{ }^{k+1}\right)
\end{aligned}
$$

Proof.

$$
\begin{aligned}
& \int_{y_{1}}^{y_{2}} \int_{x_{1}}^{x_{2}} \frac{1-y \cos x-y^{n+1} \cos (n+1) x+y^{n+2} \cos n x}{1-2 y \cos x+y^{2}} d x d y \\
& =\int_{y_{1}}^{y_{2}} \int_{x_{1}}^{x_{2}}\left(\sum_{k=0}^{n} y^{k} \cos k x\right) d x d y(\text { by Lemma } 1) \\
& =\int_{y_{1}}^{y_{2}} \int_{x_{1}}^{x_{2}} 1 d x d y+\sum_{k=1}^{n} \int_{y_{1}}^{y_{2}} \int_{x_{1}}^{x_{2}} y^{k} \cos k x d x d y \\
& =\left(x_{2}-x_{1}\right)\left(y_{2}-y_{1}\right)+ \\
& \sum_{k=1}^{n} \frac{1}{k}\left(\sin k x_{2}-\sin k x_{1}\right) \cdot \int_{y_{1}}^{y_{2}} y^{k} d y \\
& =\left(x_{2}-x_{1}\right)\left(y_{2}-y_{1}\right)+ \\
& \sum_{k=1}^{n} \frac{1}{k(k+1)}\left(\sin k x_{2}-\sin k x_{1}\right)\left(y_{2}{ }^{k+1}-y_{1}^{k+1}\right)
\end{aligned}
$$

Next, we derive the second result of this paper, and also need a lemma.

Lemma 2. The assumptions are the same as Lemma 1, then

$$
\begin{aligned}
& \frac{y \sin x-y^{n+1} \sin (n+1) x+y^{n+2} \sin n x}{1-2 y \cos x+y^{2}} \\
& =\sum_{k=1}^{n} y^{k} \sin k x
\end{aligned}
$$

Proof.

$$
\begin{aligned}
& \frac{y \sin x-y^{n+1} \sin (n+1) x+y^{n+2} \sin n x}{1-2 y \cos x+y^{2}} \\
& =\frac{(y \sin x)\left[1-y^{n+1} \cos (n+1) x\right]-(1-y \cos x) \cdot y^{n+1} \sin (n+1) x}{(1-y \cos x)^{2}+(y \sin x)^{2}} \\
& =\operatorname{Im}\left\{\frac{\left[1-y^{n+1} \cos (n+1) x\right]-i y^{n+1} \sin (n+1) x}{(1-y \cos x)-i y \sin x}\right\} \\
& =\operatorname{Im}\left\{\frac{1-y^{n+1} \cdot e^{i(n+1) x}}{1-y \cdot e^{i x}}\right\} \text { by Euler's Formula) } \\
& =\operatorname{Im}\left\{\sum_{k=0}^{n}\left(y \cdot e^{i x}\right)^{k}\right\} \\
& =\sum_{k=1}^{n} y^{k} \sin k x \text { (by Euler's Formula) }
\end{aligned}
$$

The following is our second result, we determined the closed form of the double integral (2).

Theorem 2. If the assumptions are the same as Theorem 1 , then 


$$
\begin{aligned}
& \int_{y_{1}}^{y_{2}} \int_{x_{1}}^{x_{2}} \frac{y \sin x-y^{n+1} \sin (n+1) x+y^{n+2} \sin n x}{1-2 y \cos x+y^{2}} d x d y \\
& =-\sum_{k=1}^{n} \frac{1}{k(k+1)}\left(\cos k x_{2}-\cos k x_{1}\right)\left(y_{2}{ }^{k+1}-y_{1}{ }^{k+1}\right)
\end{aligned}
$$

\section{Proof.}

$$
\begin{aligned}
& \int_{y_{1}}^{y_{2}} \int_{x_{1}}^{x_{2}} \frac{y \sin x-y^{n+1} \sin (n+1) x+y^{n+2} \sin n x}{1-2 y \cos x+y^{2}} d x d y \\
& =\int_{y_{1}}^{y_{2}} \int_{x_{1}}^{x_{2}}\left(\sum_{k=1}^{n} y^{k} \sin k x\right) d x d y
\end{aligned}
$$

(by Lemma 2)

$=\sum_{k=1}^{n} \int_{y_{1}}^{y_{2}} \int_{x_{1}}^{x_{2}} y^{k} \sin k x d x d y$

$=\sum_{k=1}^{n} \frac{-1}{k}\left(\cos k x_{2}-\cos k x_{1}\right) \cdot \int_{y_{1}}^{y_{2}} y^{k} d y$

$$
=-\sum_{k=1}^{n} \frac{1}{k(k+1)}\left(\cos k x_{2}-\cos k x_{1}\right)\left(y_{2}{ }^{k+1}-y_{1}^{k+1}\right)
$$

\section{Examples}

In the following, we aimed at the two types of double integrals we explored, to propose four examples, and use Theorems 1 and 2 to determine their solutions. On the other hand, we employ Maple to calculate the approximations of these double integrals and their closed forms for verifying our answers.

\subsection{Example 1}

By Theorem 1, we determined the following double integral

$$
\begin{aligned}
& \int_{2}^{7} \int_{\pi / 4}^{\pi / 2} \frac{1-y \cos x-y^{5} \cos 5 x+y^{6} \cos 4 x}{1-2 y \cos x+y^{2}} d x d y \\
& =\frac{5 \pi}{4}+\sum_{k=1}^{4} \frac{1}{k(k+1)}\left(\sin \frac{k \pi}{2}-\sin \frac{k \pi}{4}\right)\left(7^{k+1}-2^{k+1}\right)
\end{aligned}
$$

Using Maple to calculate the approximations of this double integral and its closed form as follows.

$>$ evalf(Doubleint $\left(\left(1-y^{*} \cos (x)-y^{\wedge} 5^{*} \cos \left(5^{*} x\right)+y^{\wedge} 6^{*} \cos \left(4^{*} x\right)\right.\right.$ )$\left.\left./\left(1-2 * y^{*} \cos (\mathrm{x})+\mathrm{y}^{\wedge} 2\right), \mathrm{x}=\mathrm{Pi} / 4 . . \mathrm{Pi} / 2, \mathrm{y}=2 . .7\right), 18\right)$;

\section{$-384.603717853869732$}

$>\operatorname{evalf}(5 * \mathrm{Pi} / 4+\operatorname{sum}(1 /(\mathrm{k} *(\mathrm{k}+1)) *(\sin (\mathrm{k} * \mathrm{Pi} / 2)-\sin (\mathrm{k} * \mathrm{Pi} / 4)) *($ $\left.\left.\left.7^{\wedge}(\mathrm{k}+1)-2^{\wedge}(\mathrm{k}+1)\right), \mathrm{k}=1 . .4\right), 18\right)$;

\section{$-384.603717853869731$}

\subsection{Example 2}

Also by Theorem 1, we can evaluate the following double integral

$$
\begin{aligned}
& \int_{-6}^{-2} \int_{\pi / 3}^{5 \pi / 6} \frac{1-y \cos x-y^{101} \cos 101 x+y^{102} \cos 100 x}{1-2 y \cos x+y^{2}} d x d y \\
& =2 \pi+\sum_{k=1}^{100} \frac{1}{k(k+1)}\left(\sin \frac{5 k \pi}{6}-\sin \frac{k \pi}{3}\right)\left[(-2)^{k+1}-(-6)^{k+1}\right]
\end{aligned}
$$

Then we use Maple to verify our answer.

$>$ evalf(Doubleint $\left(\left(1-y^{*} \cos (\mathrm{x})-\mathrm{y}^{\wedge} 101 * \cos (101 * \mathrm{x})+\mathrm{y}^{\wedge} 102 * \mathrm{c}\right.\right.$ $\left.\left.\operatorname{os}(100 * \mathrm{x})) /\left(1-2 * \mathrm{y}^{*} \cos (\mathrm{x})+\mathrm{y}^{\wedge} 2\right), \mathrm{x}=\mathrm{Pi} / 3 . .5 * \mathrm{Pi} / 6, \mathrm{y}=-6 . .-2\right), 1\right)$;

$$
-8.47342453683191052 \cdot 10^{73}
$$

$>\operatorname{evalf}(2 * \mathrm{Pi}+\operatorname{sum}(1 /(\mathrm{k} *(\mathrm{k}+1)) *(\sin (5 * \mathrm{k} * \mathrm{Pi} / 6)-\sin (\mathrm{k} * \mathrm{Pi} / 3)) *$ $\left.\left.\left((-2)^{\wedge}(\mathrm{k}+1)-(-6)^{\wedge}(\mathrm{k}+1)\right), \mathrm{k}=1 . .100\right), 18\right)$;

\section{$-8.47342453683191051 \cdot 10^{73}$}

\subsection{Example 3}

Using Theorem 2, we can obtain the following double integral

$$
\begin{aligned}
& \int_{-1 / 2}^{1 / 4} \int_{-5 \pi / 9}^{4 \pi / 9} \frac{y \sin x-y^{21} \sin 21 x+y^{22} \sin 20 x}{1-2 y \cos x+y^{2}} d x d y \\
& =-\sum_{k=1}^{20}\left[\left(\frac{1}{4}\right)^{k+1}-\left(-\frac{1}{2}\right)^{k+1}\right]
\end{aligned}
$$

Next, we employ Maple to calculate this double integral and its closed form.

$>$ evalf(Doubleint $\left(\left(\mathrm{y}^{*} \sin (\mathrm{x})-\mathrm{y}^{\wedge} 21 * \sin (21 * \mathrm{x})+\mathrm{y}^{\wedge} 22 * \sin (20 *\right.\right.$ $\left.\left.\mathrm{x})) /\left(1-2 * \mathrm{y}^{*} \cos (\mathrm{x})+\mathrm{y}^{\wedge} 2\right), \mathrm{x}=-5 * \mathrm{Pi} / 9 . .4 * \mathrm{Pi} / 9, \mathrm{y}=-1 / 2 . .1 / 4\right), 18\right)$;

$$
0.0283497926020995993
$$

$>\operatorname{evalf}(-\operatorname{sum}(1 /(\mathrm{k} *(\mathrm{k}+1)) *(\cos (4 * \mathrm{k} * \mathrm{Pi} / 9)-\cos (5 * \mathrm{k} * \mathrm{Pi} / 9)) *(($ $\left.\left.\left.1 / 4)^{\wedge}(\mathrm{k}+1)-(-1 / 2)^{\wedge}(\mathrm{k}+1)\right), \mathrm{k}=1 . .20\right), 18\right)$;

$$
0.0283497926020995995
$$

\subsection{Example 4}

Also using Theorem 2, we determined the double integral

$$
\begin{aligned}
& \int_{-2 / 3}^{1 / 6} \int_{3 \pi / 4}^{7 \pi / 4} \frac{y \sin x-y^{11} \sin 11 x+y^{12} \sin 10 x}{1-2 y \cos x+y^{2}} d x d y \\
& =-\sum_{k=1}^{10}\left[\left(\frac{1}{6}\right)^{k+1}-\left(-\frac{2}{3}\right)^{k+1}\right]
\end{aligned}
$$

We employ Maple to verify our result.

$>$ evalf(Doubleint $\left(\left(\mathrm{y}^{*} \sin (\mathrm{x})-\mathrm{y}^{\wedge} 11 * \sin (11 * \mathrm{x})+\mathrm{y}^{\wedge} 12 * \sin (10 * \mathrm{x}\right.\right.$ )$\left.\left.) /\left(1-2 * y^{*} \cos (x)+y^{\wedge} 2\right), x=3 * \operatorname{Pi} / 4 . .7 * \operatorname{Pi} / 4, y=-2 / 3 . .1 / 6\right), 18\right)$; 


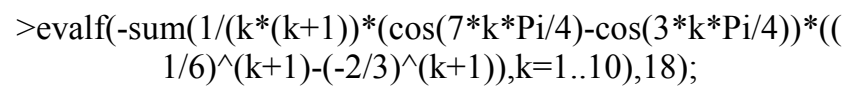

0.268559849052527921

\section{Conclusion}

As mentioned, the Euler's formula and the finite geometric series play significant roles in the theoretical inferences of this study. These two methods are different from those used in earlier works. In fact, the application of these two methods is extensive, and can be used to easily solve many difficult problems; we endeavor to conduct further studies on related applications.

On the other hand, Maple also plays a vital assistive role in problem-solving. In the future, we will extend the research topic to other calculus and engineering mathematics problems and solve these problems by using Maple. These results will be used as teaching materials for Maple on education and research to enhance the connotations of calculus and engineering mathematics.

\section{References}

[1] M. L. Abell and J. P. Braselton, Maple by Example, 3rd ed., Elsevier Academic Press, 2005.

[2] J. S. Robertson, Engineering Mathematics with Maple, McGraw-Hill, 1996.

[3] F. Garvan, The Maple Book, Chapman \& Hall/CRC, 2001.

[4] D. Richards, Advanced Mathematical Methods with Maple, Cambridge University Press, 2002.

[5] C. Tocci and S. G. Adams, Applied Maple for Engineers and Scientists, Artech House Publishers, 1996.

[6] C. T. J. Dodson and E. A. Gonzalez, Experiments in Mathematics Using Maple, Springer-Verlag, 1995.

[7] R. J. Stroeker and J. F. Kaashoek, Discovering Mathematics with Maple: An Interactive Exploration for Mathematicians, Engineers and Econometricians, Birkhauser Verlag, 1999.

[8] D. V. Widder, Advanced Calculus, Prentice-Hall, Inc., chap.
6,1961 .

[9] L. Flatto, Advanced Calculus, The Williams \& Wilkins Co., chap. 11, 1976.

[10] S, Lang, Undergraduate Analysis, Springer-Verlag, chap. 19, 1983.

[11] T. M. Apostol, Mathematical Analysis, 2nd ed., Addison-Wesley Publishing Co., Inc., chap. 14, 1975.

[12] C. H. Jr. Edwards and D. E. Penney, Calculus and Analytic Geometry, 2nd ed., Prentice-Hall, Inc, chap. 16, 1986.

[13] S. I. Grossman, Calculus, 5th ed., Saunders College Publishing, chap. 14, 1992.

[14] R. Larson, R. P. Hostetler, and B. H. Edwards, Calculus with Analytic Geometry, 8th ed., Houghton Mifflin, chap. 14, 2006.

[15] C.-H., Yu,"Application of Maple:Using the evaluation of double integrals for an example,"2013 International Conference on Intercultural Communication, Nan Jeon Institute of Technology, Taiwan, pp. 294-302, February 2013.

[16] C.-H., Yu, "Application of Maple on evaluation of four types of double integrals,"2013 Business Innovation and Development Symposium, Mingdao University, Taiwan, B20130117002, March 2013.

[17] C.-H., Yu, "Application of Maple on solving double integral problems,"2012 cross-strait Electromechanical and Industry Cooperation Conference, Ta Hwa University of Science and Technology, Taiwan, O06, November 2012.

[18] C.-H., Yu, "Application of Maple: Taking the study of two types of double integrals as an example,"SAE Seventeenth Vehicle Engineering Symposium, Nan Kai University of Technology, Taiwan, pp.856-860, November 2012.

[19] C.-H., Yu, "Application of Maple: Using the infinite series expressions of double integrals for an example," UHC2012 Sixth High Quality Family Life Key Technology Symposium, Kun Shan University, Taiwan, pp. 211-214, October 2012.

[20] C.-H., Yu,"Application of Maple: Taking the evaluation of double integrals by Fourier series as an example," ISC2012 Sixth Intelligent Application of Systems Engineering Symposium, Far East University, Taiwan, H2-6, May 2012. 